\title{
Organização, disponibilização e possibilidades de análise de dados sobre desastres de origem climática e seus impactos sobre a saúde no Brasil
}

\author{
Organization, availability and possibility of analysis of disaster data \\ of climate related origin and its impacts on health
}

Diego Ricardo Xavier ${ }^{1}$

Christovam Barcellos ${ }^{1}$

Heglaucio da Silva Barros ${ }^{1}$

Monica de Avelar Figueiredo Mafra Magalhães ${ }^{1}$

Vanderlei Pascoal de Matos ${ }^{1}$

Marcel de Moraes Pedroso ${ }^{1}$

\footnotetext{
${ }^{1}$ Instituto de Comunicação

e Informação Científica

e Tecnológica em Saúde,

Fundação Oswaldo Cruz. Av.

Brasil 4365, Manguinhos.

21.045-900 Rio de Janeiro

RJ Brasil.

diegoricardox@gmail.com
}

\begin{abstract}
The occurrence of disasters is often related to unforeseeable able natural processes. However, the analysis of major databases may highlight seasonal and long-term trends, as well as some spatial patterns where risks are concentrated. In this paper the process of acquiring and organizing climate-related disaster data collected by civil protection institutions and made available by the Brazilian Climate and Health Observatory is described. Preliminary analyses show the concentration of disasters caused by heavy rainfall events along the Brazilian coastline especially during the summer. Droughts have longer duration and extent, affecting large areas of the south and northeast regions of the country. These data can be used to analyze and monitor the impact of extreme climatic events on health, as well as identify the vulnerability and climate deteminants.

Key words Information systems, Spatial analysis, Natural disasters, Web GIS (Geographical Information Systems)
\end{abstract}

Resumo A ocorrência de desastres muitas vezes é associada a processos naturais imprevisíveis. No entanto, a análise de grandes bases de dados permite mostrar tendências sazonais e de longo prazo, bem como padrões é áreas onde se concentram riscos. Neste trabalho é descrito o processo de aquisição e organização de dados sobre desastres, coletados pelos órgãos de defesa civil, e disponibilizados pelo Observatório Nacional de Clima e Saúde. As análises preliminares mostram a concentração de eventos desastres causados por chuvas intensas ao longo da costa brasileira, principalmente durante $o$ verão. As secas apresentam maior duração e extensão, atingindo grande parte do sul e nordeste do país. Estes dados podem ser usados para analisar e monitorar o impacto de eventos climáticos extremos sobre a saúde, bem como seus determinantes de vulnerabilidade e clima.

Palavras-chave Sistemas de informação, análise espacial, desastres naturais, Web Gis 


\section{Introdução}

As grandes catástrofes evidenciam a dimensão dos impactos decorrentes de variações climáticas nos sistemas terrestres naturais e seus danos alertam a sociedade para os possíveis cenários de transformação a serem enfrentados pela humanidade. Eventos catastróficos do ponto de vista social como o furacão Katrina, que destruiu grande parte de Nova Orleans; a onda de calor na Europa em 2003, que gerou cerca de 70 mil mortes; o Catarina, primeiro furacão registrado no Atlântico Sul, que atingiu o sul do Brasil em 2004; a seca no oeste da Amazônia em 2005; as enchentes de 2009 e 2012, também na Amazônia; e os deslizamentos decorrentes das chuvas intensas na Região Serrana do Rio de Janeiro em 2011, produzindo mais de 900 mortes, são exemplos recentes de desastres relacionados a eventos climáticos extremos. Estes desastres têm gerado comoção, sofrimento e perdas, alguns com ampla cobertura midiática. Diversos outros desastres, com menor extensão ou impacto, vêm ocorrendo sem que produzam grandes repercussões políticas ou sociais. No entanto, se somados, estes pequenos desastres podem ter maior impacto que alguns grandes desastres.

No entanto, não é possível afirmar que estes eventos, vistos isoladamente, tenham sido causados pelas mudanças climáticas. É por meio do seu monitoramento, acompanhado pela análise sistemática desse conjunto de dados, que se poderá apontar tendências de mudanças de magnitude, extensão e frequência dos desastres climáticos ${ }^{1}$. Outra hipótese importante, que deve ser objeto de estudos com base em dados históricos é se está havendo um aumento, não da frequência, mas do impacto dos eventos extremos sobre a saúde, isto é, se o aumento destes impactos se deve não só às mudanças climáticas, mas também à ocupação de áreas de risco, desestruturação de sistemas de proteção social, uso inadequado do solo urbano, e outros fatores antropogênicos de aumento de vulnerabilidade ${ }^{2}$.

Entre o período de 1991 e 2010 foram registrados 31.909 desastres naturais no Brasil, com um saldo total de 2.475 mortos $^{3}$. Apenas no ano de 2011 os desastres na serra fluminense somaram mais de 900 óbitos, atingindo 3.375 pessoas. Observa-se ao longo deste período, tanto o aumento da frequência de tempestades intensas, quanto o incremento do número de pessoas afetadas direta ou indiretamente por eventos climáticos extremos ${ }^{4}$. Cabe ressaltar que o aumento do número de registros destes eventos pode ser uma consequência da melhoria dos próprios sistemas de informação, da maior repercussão dos desastres na mídia, bem como o incentivo ao reconhecimento de situações de desastre como forma de arrecadar recursos emergenciais ${ }^{3}$.

No Brasil, uma das fontes de dados sobre desastres naturais é formada pelo conjunto de decretos de situação de emergência ou estado de calamidade pública reconhecidos pelo governo federal. O critério para que se decrete situação de emergência ou estado de calamidade pública baseia-se na intensidade dos desastres, assim como na comparação entre a necessidade e a disponibilidade de recursos para o restabelecimento da situação de normalidade do município 5 . A decretação significa a ocorrência de uma situação anormal, em uma área do município, que determinou a necessidade de declarar situação de emergência ou estado de calamidade pública, para ter efeito na alteração dos processos de governo e da ordem jurídica, no território considerado, durante o menor prazo possível, para restabelecer a situação de normalidade. Emitido o decreto, deve ser realizada a descrição das características intrínsecas do desastre, da área afetada, dos danos humanos, materiais e ambientais e dos prejuízos econômicos e sociais provocados pelo desastre. Esse documento deve ser preenchido, no prazo máximo de 120 horas (5 dias) após a ocorrência do desastre, e encaminhado aos órgãos de coordenação do Sistema Nacional de Proteção e Defesa Civil (SINPDEC) ${ }^{6}$. O documento mais abrangente em relação as descrições de uma situação de desastre é o Relatótio de Avaliação de Danos (AVADAN), que, a partir da publicação na Instrução Normativa $n^{\circ} 1$, de 24/08/2012, foi substituído pelo Formulário de Informações do Desastre (FIDE). O SINPDEC bem como os instrumentos de coleta de dados sobre o desastres podem ser utilizados como subsidio ao desenvolvimento de estudos epidemiológicos, porém o acesso restrito aos dados e a baixa qualidade dos registros eram considerados como barreiras para incorporá-los em análises no âmbito da saúde ${ }^{5}$. A recente publicação dos resultados consolidados destes relatórios pode permitir sua análise sistemática e acompanhamento de tendências ${ }^{3}$.

Além dos danos imediatos oriundos dos eventos climáticos extremos, diversos problemas de saúde se estendem no período subsequente à ocorrência dos desastres naturais. Surtos e epidemias, que variam em magnitude de acordo com o grau de vulnerabilidade socioambiental e a capacidade de resiliência locais, podem aparecer, sobretudo de doenças transmissíveis. Estes outros 
dados de saúde podem ser recuperados por meio da análise de dados gerados por sistemas de informação do setor saúde, destando-se o SINAN, SIM, SIH e CNES ${ }^{5,7}$.

O Sistema de Informação de Agravos de Notificação (SINAN) é alimentado pela notificação e investigação de casos de doenças e agravos, que constam da lista nacional de doenças de notificação compulsória e compõem a base de dados do DATASUS, juntamente com outros sistemas de informação que registram eventos de saúde. Dentre eles, destacam-se o SIM (Sistema Informações de Mortalidade), que registra os óbitos segundo causas da morte, o SIH (Sistema Informações Hospitalares), que registra as internações ocorridas em hospitais próprios ou conveniados ao SUS, e CNES (Cadastro Nacional de Estabelecimentos de Saúde), que contém informações sobre a capacidade instalada dos estabelecimentos de saúde. Todos estes sistemas possuem abrangência nacional e permitem o georreferenciamento de seus dados por endereço ou código do município ${ }^{8}$.

O objetivo deste estudo é apresentar a potencialidade e o desenvolvimento de uma ferramenta de disponibilização de informações sobre desastres climáticos e seus impactos sobre a saúde, capaz de convergir dados de diversas fontes, que descrevam o impacto de eventos climáticos extremos a curto, médio e longo prazos, e auxilie no planejamento e tomada de decisões tanto frente à possibilidade de ocorrência de eventos climáticos extremos, quanto no direcionamento de programas que miniminizem os impactos humanos.

\section{Materiais e Métodos}

Este trabalho apresenta o processo de criação de um sistema de disponibilização de informações sobre desastres climáticos e seus impactos na saúde da população do Brasil, criado pelo Observatório Nacional de Clima e Saúde (www.clima saude.icict.fiocruz.br), bem como suas possibilidades de análise para a detecção de tendências temporais e padrões espaciais dos desastres e das pessoas afetadas por estes.

A primeira etapa da construção deste sistema foi a avaliação de dados disponíveis e o estabelecimento de metas e protocolos interinstitucionais. Esta etapa foi regulada pela realização de oficinas temáticas sobre os desastres climáticos e seus impactos sobre a saúde, com a participação de instituições produtoras de dados das áreas de interesse para o projeto ${ }^{7}$. Cada base de dados foi examinada segundo o grau de atualização e período coberto; a qualidade; a cobertura territorial; e os níveis de desagregação e resolução espacial. Estes critérios subsidiaram a escolha de dados que compõem o site do Observatório $\mathrm{Na}$ cional de Clima e Saúde, e o desenvolvimento de aplicativos para o acesso a estes dados.

Os gestores de instituições de saúde do SUS, pesquisadores e representantes da sociedade civil tiveram papel importante nestas oficinas, quando levantaram as principais questões institucionais e sociais envolvidas na gestão e prevenção de desastres, principalmente os relacionados à vulnerabilidade das populações aos eventos climáticos extremos. Durante as oficinas foram também abordados problemas de saúde que poderiam surgir ou se agravar em situações de desastres climáticos, o que permitiu elaborar conteúdos e funcionalidades para um sistema de disponibilização de informações voltado para o público ali representado.

\section{Organização dos bancos de dados}

Para a construção do sistema foram utilizados os dados da tabela de danos humanos, contidos nos AVADANs, no período de 2000 a 2010, disponibilizados em arquivos no formato PDF pelo Sistema Integrado de Informações sobre Desastres (S2ID) ${ }^{9}$. Os eventos foram selecionados a partir dos registros do Atlas Brasileiro de Desastres Naturais ${ }^{3}$ e do EM-DAT, organizado pelo Centre for Research on the Epidemiology of Disasters ${ }^{10}$. A complementação de eventos faltantes do banco de dados foi realizada por meio do sistema de Análise Geoespacial9 ${ }^{9}$. Todos os 10.001 registros foram digitados em planilha e importados para banco de dados SQL. Os dados de desastres foram georreferenciados segundo o município de ocorrência, fornecidos pelos instrumentos de avaliação de danos.

As informações desses instrumentos apresentam estimativas de populações diretamente atingidas, classificadas como desalojados, desabrigados, deslocados, desaparecidos, levemente feridos, gravemente feridos, enfermos, mortos e total de afetados, que inclui, além dos diretamente atingidos, todos os que sofreram algum prejuízo causado pelo desastre, como bloqueio de acessos e vias, interrupção de serviços e danos econômicos ${ }^{3}$. É importante ressaltar que se a pessoa que não se enquadra em nenhum dos danos humanos citados acima, é contada como afetada. Consequentemente, o número de pessoas afetadas não é, obrigatoriamente, o somatório dos danos humanos ${ }^{3}$. 
Além das informações contidas nos AVADANs, foram incorporados ao sistema, uma camada de informação de localização de unidades de saúde com base nas informações do CNES (Cadastro Nacional de Estabelecimentos de Saúde), Também foram acrescentados dados de doenças de notificação compulsória (dengue, hepatite A e leptospirose), contidas no SINAN (Sistema Nacional de Agravos de Notificação), e dados de mortalidade do SIM (Sistema de Informação de Mortalidade). Segundo a classificação da CID-10, os códigos X30 a X39 contemplam exposição às forças da natureza: (X30) Exposição a calor natural excessivo, (X31) Exposição a frio natural excessivo, (X32) Exposição à luz solar, (X33) Vítima de raio, (X34) Vítima de terremoto, (X35) Vítima de erupção vulcânica, (X36) Vítima de avalanche, desabamento de terra e outros movimentos da superfície terrestre, (X37) Vítima de tempestade cataclísmica, (X38) Vítima de inundação, (X39) Exposição a outras forças da natureza e às não especificadas.

O sistema de disponibilização de dados sobre desastres é baseado na concatenação de códigos elaborados em campos usados como chave-estrangeira na tabela de eventos extremos, facilitando, dessa forma, a identificação das informações básicas dos eventos em uma consulta. A ferramenta de consulta das informações foi desenvolvida usando linguagem SQL (Structured Query Language), e o sistema foi desenvolvido com software livre, utilizando várias linguagens de programação (html, php, ajax, javascript, entre outros).

A interface do sistema (Figura 1) foi criada de forma a facilitar o manuseio por todos os ní-

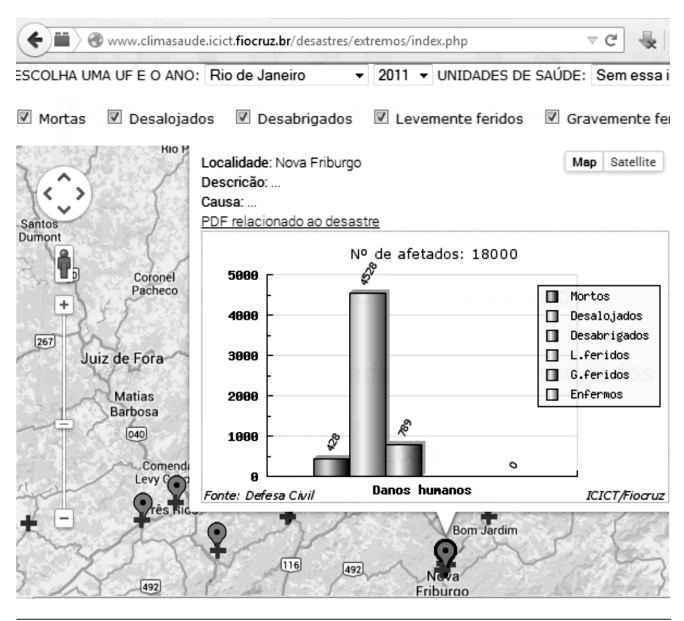

Figura 1. Interface do Sistema de Eventos Extremos no Brasil. veis de usuários. Para isso inicia-se sempre com a seleção de uma unidade da federação (UF) e um ano, como também a localização das unidades de saúde segundo sua especialidade. Os dados disponíveis na base do CNES contêm os endereços de estabelecimentos de saúde e, por meio da ferramenta wget, distribuída pelo projeto $\mathrm{GNU}^{11}$ foi possível adquirir e organizar seus dados em tabelas de relacionamento simples, para realizar o processo de georreferenciamento. Por meio de algoritmos desenvolvidos em linguagem JAVA, as informações relativas aos endereços são "varridas" e georreferenciadas a partir das possibilidades do conjunto de endereços disponíveis na base do Google Maps. Contudo, devido aos riscos inerentes ao mau preenchimento do campo de endereço da base do CNES, optou-se pelo uso de alguns mecanismos de segurança para confirmação do georreferenciamento como, por exemplo, o índice de similaridade fonética, que compara trechos de variáveis textuais (strings) com seus fonemas, que, embora apresentem escritas diferentes, podem servir de conexão entre as bases durante o processo de georreferenciamento ${ }^{12}$. Outro recurso utilizado para diminuir os erros, foi a janela de restrição, que consiste em verificar se as coordenadas encontradas estão inseridas dentro dos limites do município para as quais foram destinadas e averiguar se o endereço escrito na ficha do CNES difere do endereço encontrado na base. Em seguida, o usuário pode selecionar qual restrição por tipo de atingido (Mortas, Desalojadas, Desabrigados, Levemente feridos, Gravemente feridos ou Enfermos) será relacionada nas tabelas, mapas e gráficos.

Para construção do visualizador de mapas, foram utilizadas interfaces programáveis fornecidas pela Google, que através das possibilidades de customização contidas na ferramenta, os dados passam a ser representados por marcadores de pontos na base do Google Maps. Esta personalização é adaptada para atender ao conjunto de dados de saúde e eventos extremos, gerando mapas interativos de exibição web ${ }^{13}$.

O sistema de eventos extremos oferece como mecanismo de visualização a técnica de análise exploratória, mais especificamente a análise de padrões de pontos: o estimador de intensidade (Kernel estimation). Este tipo de análise espacial segundo Druck et al. ${ }^{14}$ pode ser utilizado de forma simples, apenas com base na análise visual do padrão de distribuição de um conjunto de eventos no mapa. Com este tipo de análise aplicada no módulo de mapas, adicionando marcadores de intensidade às localizações dos eventos extre- 
mo,s foi possível definir qual a extensão territorial dos eventos.

Todas as informações estão disponibilizadas no site do Observatório Nacional de Clima e Saúde ${ }^{7}$.

\section{Análises temporais e espaciais}

Com base nas informações disponibilizadas pelo Observatório Nacional de Clima e Saúde, foram realizadas análises de séries temporais e análises espaciais, tendo como variáveis dependentes a frequência dos desastres e número de pessoas afetadas. Para realização das análises temporais e espaciais, as causas de eventos climáticos foram distribuídas em seis grupos segundo a Codificação de Desastres, Ameaças e Riscos (Codar) (Quadro 1).

As séries históricas foram analisadas a fim de se verificar períodos em que ocorreu o maior número de afetados, bem como os tipos de causas. Foram construídos gráficos com componentes de tendência e sazonalidade, além da série de dados de afetados. O componente "tendência" foi utilizado para ajudar a identificar ciclos sazonais e plurianuais de ocorrência na amplitude de afe- tados. O componente sazonal é importante para observação da ocorrência de ciclos interanuais. Para análise das séries temporais foi utilizado o software R 3.0.2 ${ }^{15}$.

Para a análise espacial, foi utilizada a estimativa de Kernel, levando-se em consideração os centróides (centro geográfico) dos municípios. Os estimadores Kernel utilizam uma função de ponderação mais complexa do que a média simples ou o inverso do quadrado da distância. Essa classe de estimadores é descrita na literatura ${ }^{16,17}$ como estimadores de densidade não-paramétricos e generalizam a ideia sobre as médias móveis locais, ao supor que a densidade dos fenômenos varia localmente de forma suave, sem "picos" nem "descontinuidades". Seu objetivo é produzir superfícies de dados mais suaves, que são mais representativas (no espaço) de fenômenos naturais e socioeconômicos.

Em resumo, os estimadores Kernel são uma alternativa viável a métodos mais sofisticados de interpolação, pois não requerem a parametrização da estrutura de correlação espacial, como no caso de diversas técnicas de geoestatística. As desvantagens destes estimadores são a forte de-

Quadro 1. Soma da população atingida para os eventos disponibilizados no sistema segundo causas de eventos utilizadas neste estudo e tipo de dano sofrido.

\begin{tabular}{|c|c|c|}
\hline Causas & CODAR & Evento \\
\hline Eólicas & $\begin{array}{l}12101 \\
12102 \\
12103 \\
12104\end{array}$ & $\begin{array}{l}\text { Vendavais ou Tempestades } \\
\text { Vendavais Muito Intensos ou Ciclones Extratropicais } \\
\text { Vendavais Extremamente Intensos, Furacões, } \\
\text { Tufões ou Ciclones Tropicais } \\
\text { Tornados e Trombas d'Águas }\end{array}$ \\
\hline Granizo/Geadas & $\begin{array}{l}12201 \\
12205 \\
12206\end{array}$ & $\begin{array}{l}\text { Ondas de Frio Intenso } \\
\text { Granizo } \\
\text { Geadas }\end{array}$ \\
\hline Inundação Brusca & 12301 & Enchente ou inundação gradual \\
\hline Inundação Gradual & $\begin{array}{l}12302 \\
12303\end{array}$ & $\begin{array}{l}\text { Enchente ou inundação brusca } \\
\text { Alagamentos }\end{array}$ \\
\hline Secas & $\begin{array}{l}12401 \\
12402 \\
12404\end{array}$ & $\begin{array}{l}\text { Estiagens } \\
\text { Secas } \\
\text { Incêndios }\end{array}$ \\
\hline Deslizamentos & $\begin{array}{l}13301 \\
13302 \\
13303 \\
13309\end{array}$ & $\begin{array}{l}\text { Escorregamentos ou Deslizamentos } \\
\text { Corridas de Massa } \\
\text { Rastejos } \\
\text { Erosão Marinha }\end{array}$ \\
\hline
\end{tabular}


pendência do raio de busca e, em alguns casos, a excessiva suavização da superfície que pode esconder variações locais importantes.

A interpolação de Kernel foi gerada com o objetivo de identificar áreas de maior concentração (densidade) de eventos e de pessoas afetadas com base nos seguintes parâmetros: grade de 200 colunas sobre a região estudada, com algoritmo de função quártica e raio adaptativo. O software utilizado na elaboração dos mapas foi o TerraView $4.2^{18}$.

\section{Resultados}

O sistema de desastres naturais e impactos a saúde do Observatório Nacional de Clima e Saúde apresenta mapas de pontos segundo eventos, destacando-se ocorrências em que houve óbitos. Ao selecionar um ponto do mapa, abre-se uma janela com todas as informações do evento e um gráfico que exibe os danos humanos. Fazem parte do resultado da consulta gráficos que mostram: (i) a soma do total de todos os danos humanos dos eventos exibidos no mapa; (ii) a distribuição dos eventos durante o ano, o que facilita a visualização do componente de sazonalidade; (iii) os agravos distribuídos nos meses, obtidos do SINAN, que podem ter relação com os eventos; além de tabulações dos danos humanos e dos eventos por municípios de ocorrência, ordenados pelo maior número de óbitos (Figura 1). Os estabelecimentos de saúde são visualizados por meio de símbolos em forma de cruz em vermelho como uma camada de informações sobre a base de mapas. O usuário pode optar pela visualização das unidades de saúde segundo o nível de complexidade do atendimento e UF de seleção. É possível se ter informações relacionadas ao esta- belecimento de saúde, como nome, localização e complexidade de atendimento, clicando no ícone referente a unidade que aparece no mapa. É possível também desabilitar essa função e visualizar apenas os eventos no mapa.

A Tabela 1 apresenta a soma da população atingida para os eventos disponibilizados no sistema segundo causas de eventos utilizadas neste estudo e tipo de dano sofrido. A maior parte dos desastres tem origem em eventos climáticos, principalmente mediados pela água, que por excesso ou carência podem provocar danos materiais, doenças e óbitos. Este padrão, ao contrário de outros países sujeitos também a eventos de origem tectônica, coloca o Brasil em uma situação particular, mais afetado pelo clima e suas variações.

A maior letalidade foi observada para os desastres envolvendo deslizamentos, que apresentaram 91 óbitos para cada 100.000 afetados, seguidos das inundações, com 3 óbitos para cada 100.000 afetados. Outros tipos de desastres apresentaram uma baixa letalidade, com menos que 1 óbito para cada 100.000 afetados. Por outro lado, são os eventos eólicos, e principalmente os granizos, que provocam o maior proporção de desalojados e desabrigados, cerca de $13 \%$ do total de afetados. Apesar da grande frequência e extensão dos eventos de seca, os impactos sobre a saúde são os menores entre os tipos de desastres. Este resultado é provavelmente subestimado, já que as estimativas de desalojados, desabrigados, mortos e feridos realizados pela Defesa Civil não consideram os efeitos indiretos e a longo prazo deste tipo de desastre. As secas costumam gerar danos indiretos como a fome, a interrupção de suprimento de água e alimentos, contaminação de fontes de água, perdas econômicas, desemprego, migrações, entre outros fatores que impactam a saúde ${ }^{19}$. Se considerados estes efeitos indiretos,

Tabela 1. Soma da população atingida para os eventos disponibilizados no sistema segundo causas de eventos utilizadas neste estudo e tipo de dano sofrido.

\begin{tabular}{lrrrrrr}
\hline & Eólica & Granizo & $\begin{array}{c}\text { Inundações } \\
\text { Graduais }\end{array}$ & $\begin{array}{c}\text { Inundações } \\
\text { bruscas }\end{array}$ & Secas & Deslizamentos \\
\hline Desalojados & 136.135 & 86.379 & 941.824 & 1.155 .391 & 19.292 & 47.923 \\
Desabrigadas & 28.032 & 8.459 & 326.895 & 329.998 & 2.932 & 29.168 \\
Deslocadas & 8.795 & 12.056 & 99.027 & 199.126 & 131.805 & 4.097 \\
Desaparecidas & 3 & 2 & 150 & 759 & 3.350 & 17 \\
Levemente Feridas & 1.925 & 928 & 9.821 & 27.089 & 430 & 1.130 \\
Gravemente Feridas & 101 & 68 & 545 & 689 & 68 & 53 \\
Enfermas & 773 & 1.366 & 116.704 & 99.507 & 49.683 & 552 \\
Mortas & 17 & 2 & 270 & 615 & 180 & 1.221 \\
Afetadas & 2.495 .523 & 697.132 & 7.612 .614 & 23.041 .201 & 36.418 .636 & 1.333 .606 \\
\hline
\end{tabular}


o impacto das secas sobre a mortalidade pode ser maior que os de outros tipos de desastres, de impactos mais evidentes e imediatos ${ }^{20}$.

Em algumas situações existem discordâncias das informações quanto ao preenchimento dos instrumentos de avaliação de danos. As instruções de preenchimento do documento apontam que pessoas que não se enquadram em nenhum dos danos humanos, são contadas como afetadas. Entretanto, existem registros que apresentam o preenchimento das demais variáveis de danos humanos e ausência de preenchimento da variável afetados. Essa situação provoca falta de alguns instrumentos, quando analisados o número de decretos segundo afetados. Em outras palavras foram contabilizados no sistema 10.001 instrumentos de avaliação de danos, mas apenas 9.426 apresentam o preenchimento da variável afetados. Foram observadas outras inconsistências como os valores registrados para desaparecimentos. Por exemplo, em Itauaçu (BA) foram registrados em maio de 2003, 1.550 desaparecidos durante uma seca. Provavelmente este valor indica o número de deslocados. Esses e outros equívocos oriundos do preenchimento inadequado do AVADAN foram verificados na elaboração do banco. Para conclusão final do banco de dados outros documentos, como informações da imprensa, foram utilizados para consolidação das informações.

A Tabela 2 mostra a comparação entre o número de afetados segundo causas dos desastres climáticos e número de eventos. O maior número de eventos é observado nas secas com $49 \%$ do total de eventos registrados, em seguida são observadas as inundações bruscas com 31\%. O percentual de afetados também é maior em eventos de secas com 52\% dos afetados, as inundações bruscas apresentam cerca de $28 \%$, nas inundações graduais e em causas eólicas ocorre um maior percentual de afetados em um menor número de eventos. Apesar de um número menor de eventos em comparação com as demais causas, a média de atingidos pelos deslizamentos é a maior observada, com mais de 115 mil pessoas afetadas em cada desastre.

$\mathrm{O}$ evento em que ocorreu maior número de afetados foi uma inundação brusca que atingiu o município de Salvador na Bahia, no ano de 2011, com 1.431.793 pessoas afetadas, cerca de metade da população do município. Apesar do elevado número de afetados não foram notificados óbitos pela defesa civil. O maior número de afetados pela seca foi uma estiagem ocorrida nos anos de 2002 e 2008 em Chapecó, Santa Catarina, quando 169.912 pessoas foram afetadas. Em Teresina, no estado do Piauí, ocorreu em abril de 2009 um evento de inundação gradual que atingiu 779.939 pessoas, cerca de $94 \%$ da população do município. No município de Criciúma em março de 2004, ocorreu evento eólico com o maior número de afetados: 120.000 pessoas. Os eventos de deslizamentos contabilizaram 1.333 .606 pessoas afetadas no período de estudo, e o município de Ponta Grossa, no Paraná, foi o que apresentou o maior número de afetados, 240.000 no mês de julho de 2011, o que representa em torno de $77 \%$ da população do município.

A Figura 2 apresenta a série histórica do número de afetados mensalmente segundo grupos de causas de desastres naturais no período de 2000 a 2011. A Figura 2.1 apresenta a série histórica de eventos de causas eólicas. Observa-se um valor extremo no mês março de 2004, que corresponde à passagem do furacão Catarina, e, no mês de maio de 2008, que coincide com um ciclone que atingiu municípios de Santa Catarina e Rio Grande do Sul. O fenômeno é frequente na região e, devido a um bloqueio atmosférico, o ciclone permaneceu próximo a costa. No ano de 2009 , foram atingidos cerca de 70 municípios do Rio Grande do Sul. Ventos de mais de $113 \mathrm{~km} / \mathrm{h}$ atingiram o estado, provocando muitos estragos na região.

Tabela 2. Comparação entre o número de afetados segundo causas dos desastres climáticos e número de eventos.

\begin{tabular}{lrrrrrr}
\hline Causa do desastre & Eventos & $\begin{array}{c}\text { Máximo } \\
\text { afetados }\end{array}$ & $\begin{array}{c}\text { Soma } \\
\text { afetados }\end{array}$ & $\begin{array}{c}\text { Média } \\
\text { afetados }\end{array}$ & $\begin{array}{r}\text { Percentual } \\
\text { afetados }\end{array}$ & $\begin{array}{r}\text { Percentual } \\
\text { de eventos }\end{array}$ \\
\hline Eólicas & 477 & 120.000 & 2.495 .523 & 5.231 & 3.39 & 5.06 \\
Granizo / Geadas & 306 & 50.000 & 697.132 & 2.278 & 0.95 & 3.25 \\
Inundações Graduais & 1.205 & 779.939 & 7.612 .614 & 6.317 & 10.33 & 12.78 \\
Inundações Bruscas & 2.680 & 1.431 .793 & 23.041 .201 & 8.597 & 31.28 & 28.43 \\
Secas & 4.643 & 169.912 & 36.418 .636 & 7.843 & 52.24 & 49.26 \\
Deslizamentos & 115 & 240.000 & 1.333 .606 & 11.596 & 1.81 & 1.22 \\
\hline
\end{tabular}



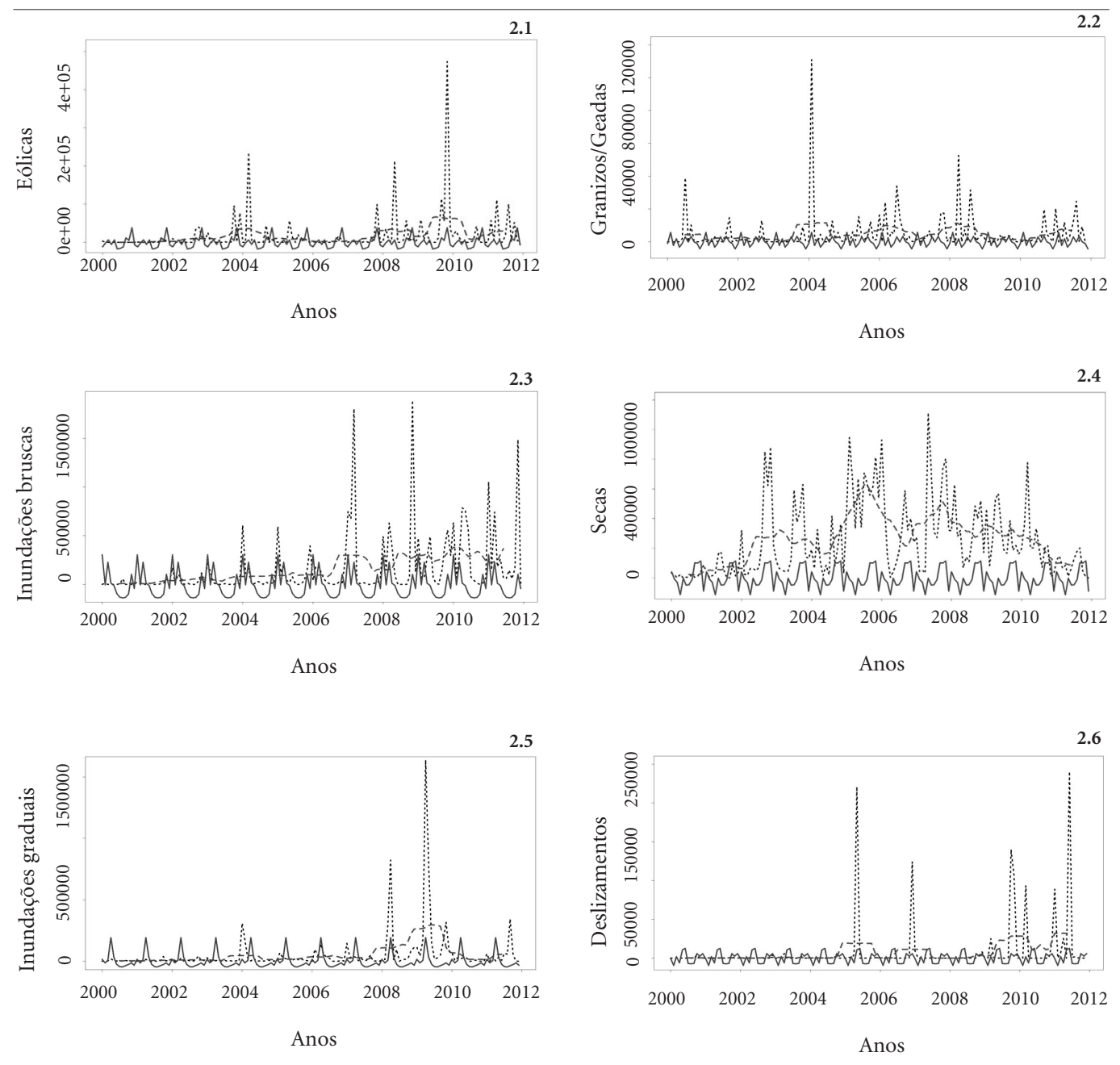

...-..- Afetadas ...-. Tendência — Sazonalidade

Figura 2. Série histórica do número de afetados mensalmente segundo grupos de causas de desastres naturais no período de 2000 a 2011.

Os eventos relacionados a granizos e geadas são apresentados na Figura 2.2. Foi observado no ano de 2004 o maior volume de atingidos, como consequência de eventos ocorridos em fevereiro e março, resultado da passagem de jato subtropical com forte intensidade e a presença de sistemas de baixa pressão. Nos casos analisados, verificouse que os eventos de grande intensidade foram potencializados por mudanças bruscas de temperatura na superfície, associadas a quantidades significativas de água precipitável na atmosfera ${ }^{21}$.

Os eventos de inundações bruscas na Figura 2.3 apresentam um comportamento sazonal bem definido e reflete as temporadas de chuvas ocorridas durante os períodos de verão no país. Além disso, é observada uma tendência de aumento do número de afetados nos últimos anos da série. Em 2004 os municípios mais atingidos se situavam na Região Sudeste. Em 2008, mais de 300 mil pessoas foram atingidas por inundações bruscas no Nordeste durante os meses de março e abril e fortes chuvas no sul do país durante o fim do ano. Em 2010, chuvas intensas causaram inundações bruscas em municípios de Alagoas e Pernambuco. No ano seguinte, fortes chuvas atingiram o Sudeste e Sul do Brasil principalmente o município de Blumenau em Santa Catarina.

As secas e estiagens (Figura 2.4) apresentam comportamentos cíclicos bienais que podem estar relacionados com as secas ocorridas no 
Nordeste e períodos de estiagens no sul do país. Os meses de março, abril, maio e junho correspondem ao trecho descendente da curva com períodos de estiagem ocorrendo principalmente no Sul do país. Os meses de agosto setembro, outubro e novembro, em geral descrevem períodos em que ocorrem os decretos de seca no Nordeste do Brasil e se situam no trecho ascendente da curva, segundo a linha de tendência.

As inundações graduais (Figura 2.5) apresentaram elevados números de atingidos em abril de 2008, quando rios de municípios do Nordeste brasileiro e municípios do Pará tiveram elevação das águas. Também no vale do Rio Itajaí, em Santa Catarina, ocorreu neste ano uma das maiores inundações já registradas no país. Em 2009, foram registradas mais de 1 milhão e 600 mil pessoas afetadas devido a inundações graduais, em áreas da Amazônia, Nordeste e Sudeste brasileiros.

A maioria dos deslizamentos e escorregamentos (Figura 2.6) ocorreram nos municípios da regiões Sudeste e, eventualmente, em áreas do Sul do país. No mês de maio de 2005 ocorreram deslizamentos no município de Olinda, onde cerca de 220 mil pessoas foram atingidas. Em janeiro de 2007, municípios do estado de Minas Gerais e Rio de Janeiro foram atingidos por deslizamentos. Nos últimos meses do ano de 2009 e início de 2010, municípios do estado do Rio de Janeiro sofreram deslizamentos na região litorânea e serrana do estado. Em 2011 ocorreu o maior desastre da história do país, com mais de 900 óbitos e mais de 240 mil afetados na região serrana do estado.

A Figura 3 mostra a densidade de eventos e de pessoas afetadas por desastres, obtida pela interpolação de dados pelo método de estimadores kernel, segundo grupos de eventos. De um modo geral, há uma grande concentração de desastres ao longo da costa sul e sudeste (Figuras 3a e 3b), sujeita a entrada de frentes frias e complexos convectivos, que desencadeiam chuvas intensas e concentradas na região ${ }^{4}$.

Observa-se que a distribuição espacial dos eventos se distingue pela natureza do evento. Deste modo, os efeitos dos eventos eólicos (ventos, ciclones, tufões, etc.) atingem com maior intensidade o Sul do país (Figuras 3c e 3d). A chuva de granizo afeta principalmente as áreas de planalto da região Sul (Figuras $3 e$ e $3 \mathrm{f}$ ). As inundações afetam mais pessoas nas regiões Sul, Sudeste e Nordeste (Figuras 3h e 3j), sendo também observadas no interior destas regiões, em áreas afastadas do oceano. Já os deslizamentos estão concentrados ao longo da faixa litorânea,
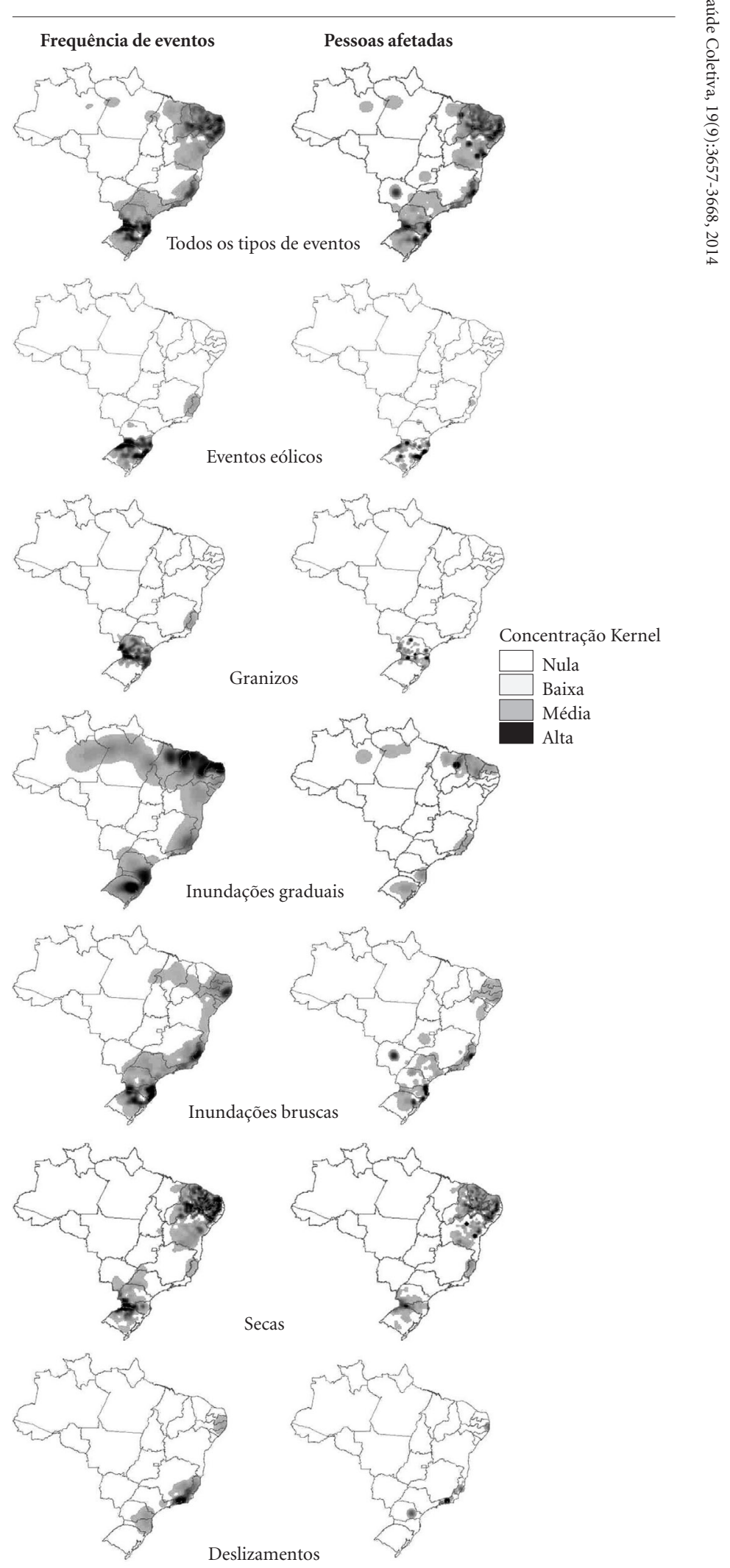

Figura 3. Mapeamento das densidades Kernel para número de eventos e o total de pessoas afetadas, por grupos de desastres.

Fonte: Elaborado pelos autores com dados georreferenciados dos AVADANS - Sistema Nacional de Defesa Civil (SINDEC). 
principalmente em áreas de encosta, como a Serra do Mar (Figuras 3m e 3n).

É importante verificar que algumas áreas estão sujeitas a uma sobreposição de riscos, sendo atingidas tanto por secas quanto por inundações. Este é o caso, por exemplo, da região Nordeste, onde as secas (Figuras 3k e 3l) e períodos de chuvas intensas se intercalam. Já a Amazônia ocidental, ao longo da calha principal do Rio Amazonas, vem registrando períodos de cheias seguidas de secas (Figura 3g), demonstrando uma tendência de aumento na amplitude do nível dos rios. Este padrão de variação, que supera os limites esperados da variação sazonal, pode ser consequência de mudanças climáticas ${ }^{22}$ e já vem provocando danos à vida de habitantes locais, dificultando o transporte e o abastecimento de água e alimentos nos períodos de seca, ou inundando partes das cidades e localidades ribeirinhas em períodos de cheia $^{23,24}$.

A comparação entre os mapas contendo as densidades de ocorrência de eventos (Figura 3a) com a densidade de pessoas afetadas (Figura $3 b$ ) mostra uma concentração maior dos impactos nas áreas de maior densidade demográfica e que podem ser consideradas como mais vulneráveis. As vulnerabilidades das populações e do território podem explicar a diferença na distribuição espacial verificada entre os locais atingidos pelos desastres e as áreas com maior número de feridos e mortos durante estes desastres, que nem sempre são coincidentes ${ }^{25}$.

\section{Discussão}

O georreferenciamento e a disponibilização de dados em forma de mapas permitem recuperar o contexto em que os desastres climáticos são produzidos. As tabelas ou mapas por UF se mostram pouco eficazes na identificação de áreas de risco porque estas áreas diferem grandemente do desenho de unidades administrativas. Os limites das áreas de risco se assemelham muito mais aos biomas ou unidades geomorfológicas, como por exemplo os deslizamentos, que afetam principalmente o litoral de Mata Atlântica, as secas que atingem com mais frequência o semiárido nordestino e o interior da região sul. $\mathrm{O}$ exame do conjunto de dados sobre desastres permite reconhecer padrões de distribuição temporal e espacial, bem como seus possíveis determinantes sociais, ambientais e climáticos. A repetição de eventos em uma mesma região, mesmo que de menor extensão e impacto, é um marcador de risco para situações futuras. Alguns padrões que podem ser adquiridos deste conjunto de eventos são a magnitude, extensão e frequência de eventos por tipologia, número de afetados, etc. Ao mesmo tempo, a concentração destes eventos em algumas áreas constitui uma pista para identificar vulnerabilidades socioambientais, como a declividade nos casos de deslizamentos, o clima no caso das secas, entre outros. Deste modo, a vulnerabilidade não pode ser considerada como um fator único e universal, já que o mesmo evento climático pode produzir maiores danos em algumas áreas e sobre alguns grupos sociais com maior intensidade. Do mesmo modo, a natureza do evento pode afetar mais algumas áreas que outras, o que pode ser observado pela distribuição desigual dos riscos nos mapas produzidos ao nível nacional. Um exemplo é o efeito da seca, que no sul promove a perda da produção agrícola e afeta uma grande quantidade pessoas. No nordeste, a seca tem efeitos mais severos sobre as condições de sobrevivência de pessoas, podendo acarretar no adoecimento, subnutrição, sofrimento e morte, com maior intensidade.

É importante destacar que os impactos de eventos climáticos extremos sobre a saúde são bastante diferenciados, dependendo da vulnerabilidade dos grupos populacionais, sua capacidade de adaptação e resiliência. Obviamente, diferentes populações vivendo em espaços diferenciados apresentam distintas constituições para suas vulnerabilidades, entendida como a capacidade de resposta de indivíduos, grupos e comunidades aos potenciais perigos deflagrados por eventos relacionados a mudanças climáticas e ambientais ao longo dos processos saúde-doença.

Segundo esta concepção, a vulnerabilidade destas populações está associada aos territórios de sua vida cotidiana, e sua caracterização não pode prescindir de uma visão ecológica, institucional e social ${ }^{26}$. Observar a distribuição espacial da população e sua dinâmica, os indicadores locais de pobreza e desigualdades, a estrutura institucional de proteção, bem como a dinâmica dos ecossistemas em que estão inseridos, são alguns dos componentes que permitirão descrever, medir e acompanhar a vulnerabilidade em saúde de grupos populacionais quanto aos potenciais cenários climáticos futuros e preparar o setor saúde para o seu enfrentamento. Segundo Vera et al. ${ }^{27}$, os principais desafios para a disseminação de dados sobre clima são: a construção de parcerias entre gestores, usuários e sociedade civil e os produtores de dados sobre clima; a tradução de dados de longo prazo em informações na 
escala regional e local, de acordo com os níveis de decisão; manutenção de um sistema global de observação do clima; e procedimentos para a integração, avaliação da qualidade, processamento e análise de bases de dados relevantes para a predição do clima. Neste sentido, o debate sobre as mudanças ambientais e climáticas globais e seus impactos sobre as condições de saúde deve permitir estabelecer canais de comunicação entre produtores e usuários de dados, sendo os primeiros compreendidos como instituições que possuem entre suas atribuições a coleta e divulgação de dados de forma sistemática. Já os segundos são constituídos por pessoas ou grupos interessados na resposta que estes dados podem trazer para suas indagações, o que depende de seus interesses e sua capacitação técnica.

Este estudo tem limitações, sobretudo quando observadas as bases de dados utilizadas. Os registros de desastres costumam conter erros de omissão, tipologia e quantificação dos danos e prejuízos ${ }^{4}$. Neste sentido, o uso destas bases de dados deve ser encarado, também, como uma possibilidade de análise, utilizando uma fonte de dados, mesmo que com limitações de preenchimento, decorrentes de treinamentos diferenciados das equipes das defesas civis, estaduais, displicência no preenchimento de alguns instrumentos de avaliação de danos, e reestruturação constante da base de dados. A defasagem quanto à publicação dos dados pela defesa civil é a informação mais importante durante um evento de catástrofe natural. Como todo sistema de informação, este tem apresentado melhoras, evoluindo para um sistema mais dinâmico. De todo modo, as análises aqui apresentadas subsidiam um melhor planejamento de estratégias de adaptação e mitigação frente a eventos climáticos extremos que, apesar de estocásticos, apresentam componentes temporais e espaciais que conformam padrões, passíveis de serem previstos, monitorados e consequentemente terem seus danos atenuados.

A sistematização destes dados sobre desastres tem-se apresentado como uma maneira eficaz de se identificar a ocorrência de desastres naturais, especialmente os de baixa intensidade, que apesar de não levarem os municípios a decretar situação de emergência, podem causar prejuízos materiais e humanos à sociedade. A coleta e disponibilização de dados por meio dos instrumentos do SINPDEC pode suprir a falta de registros históricos sobre desastres naturais no Brasil, permitindo, a longo prazo, a formação de um importante banco de dados sobre desastres naturais. Ao mesmo tempo, deve-se empreender um esforço para padronizar os termos e metodologias de coleta de dados, o que pode influir na qualidade dos dados sobre desastres e distorcer avaliações sobre a sua magnitude e tendências ${ }^{28}$.

Outras funcionalidades podem ser agregadas a estes sistemas que combinam mapas na web, acesso a bancos de dados geográficos e a coleta de dados espaciais, voluntária e distribuída ${ }^{29}$. Neste caso, a Internet adquire papel relevante não só como ferramenta para divulgação e disseminação destas informações, mas também como meio para a produção de informações ${ }^{30}$. Estes sistemas, como a plataforma Ushahidi têm sido utilizados para o gerenciamento de recursos em situações de desastres, como no terremoto do Haiti e do Chile em 2010. A crescente disponibilidade de aparelhos de telecomunicação móveis com funções de posicionamento e trocas de mensagens (SMS), bem como a participação em redes sociais virtuais, como Facebook e Twitter, pode facilitar a formação de uma rede de cidadãos-sensores, que alimentam e utilizam dados geográficos de interesse comum ${ }^{31}$. Obviamente, esta nova configuração de sistemas deve ser adaptada ao contexto brasileiro, no qual não existe cobertura para serviços de telecomunicação móvel em grande parte do território nacional, principalmente em áreas rurais e remotas, e o acesso a equipamentos de telefonia móvel (smartphones) é ainda restrito para parcelas mais pobres da população. Estas desigualdades sociais, que se manifestam no espaço, podem produzir vieses de coleta e análise de dados, promovendo falsos alertas em áreas mais ricas e apagando as reais necessidades das parcelas mais carentes da população, principalmente em situações de emergência.

\section{Colaboradores}

DR Xavier, C Barcellos, HS Barros, MAFM Magalhães, VP Matos e MM Pedroso participaram igualmente de todas as etapas de elaboração do artigo. 


\section{Referências}

1. El Morjani Zel A, Ebener S, Boos J, Abdel Ghaffar E, Musani A. Modelling the spatial distribution of five natural hazards in the context of the WHO/EMRO Atlas of Disaster Risk as a step towards the reduction of the health impact related to disasters. Int J Health Geogr. 2007; 7(6):8.

2. Tompkins EL, Lemos MC, Boyd E. A less disastrous disaster: Managing response to climate-driven hazards in the Cayman Islands and NE Brazil. Global Environmental Change 2008; 18(4):736-745.

3. Centro Universitário de Estudos e Pesquisas sobre Desastres. Atlas brasileiro de desastres naturais 1991 a 2010: volume Brasil. Florianópolis: CEPED,UFSC; 2012.

4. Marcelino EV, Nunes LH, Kobiyama M. Banco de dados de desastres naturais: análise de dados globais e regionais. Caminhos de Geografia. 2006; 6(19):130-149.

5. Sobral A, Machado CF, Desastres naturais - sistemas de informação e vigilância: uma revisão da literatura. Epidemiol. Serv. Saúde. 2010; 19(4):389-402.

6. Brasil. Presidência da República. Casa Civil. Subchefia para Assuntos Jurídicos. Lei No 12.608, de 10 de abril de 2012. Institui a Política Nacional de Proteção e Defesa Civil - PNPDEC; dispõe sobre o Sistema Nacional de Proteção e Defesa Civil - SINPDEC e o Conselho Nacional de Proteção e Defesa Civil - CONPDEC; autoriza a criação de sistema de informações e monitoramento de desastres; altera as Leis nos 12.340 , de 10 de dezembro de 2010, 10.257, de 10 de julho de 2001, 6.766, de 19 de dezembro de 1979, 8.239, de 4 de outubro de 1991, e 9.394, de 20 de dezembro de 1996; e dá outras providências. Diário Oficial da União 2012; 11 abr.

7. Observatório Nacional de Clima e Saúde. Relatório da oficina sobre desastres e eventos climáticos extremos. Rio de Janeiro: Fiocruz; 2010.

8. Barcellos C, Ramalho WM, Gracie R, Magalhães MAFM, Fontes MP, Skaba D. Georreferenciamento de dados de saúde na escala submunicipal: algumas experiências no Brasil. Epidemiol. Serv. Saúde 2008; 17(1):59-70.

9. Secretaria Nacional de Defesa Civil. Sistema Integrado de Informações sobre Desastres - S2ID. [acessado 2013 out 12]. Disponível em: http://s2id.integracao.gov.br/ relatorio/geoespacial/geoespacial.html.

10. CRED. The OFDA/CRED International Disaster Database. [on line]. [acessado 2013 out 12]. Disponível em: http://www.em-dat.net/

11. Ma X, Liberman M. Bits: A method for bilingual text search over the web. Machine Translation Summit VII. 1999; p. 538-542.

12. Holmes D, McCabe MC. Improving precision and recall for soundex retrieval. In: Proceedings Information Technology: Coding and Computing, 2002. International Conference on. IEEE; 2002. p. 22-26.

13. Boulos M. Web GIS in practice III: creating a simple interactive map of England's strategic Health Authorities using Google Maps API, Google Earth KML, and MSN Virtual Earth Map Control. Int J Health Geogr 2005; 4(1):22.

14. Druck S, Carvalho MS, Câmara G, Monteiro AMV. Análise Espacial de Dados Geográficos. Planaltina: Embrapa; 2004.

15. R: A language and environment for statistical computing. R Foundation for Statistical Computing, Vienna, Austria; 2013. Disponível em: http://www.R-project.org/
16. Bailey T, Gattrel AC. Interactive Spatial Data Analysis. London: Longman; 1995.

17. Casanova M, Câmara G, Davis C, Vinhas L, Queiroz GR. Bancos de dados geográficos. Curitiba: MundoGEO; 2005.

18. Instituto Nacional de Pesquisas Espaciais (INPE). Programa Terraview versão 4.2. São José dos Campos: INPE; 2010.

19. Stanke C, Kerac M, Prudhomme C, Medlock J, Murray V. Health effects of drought: a systematic review of the evidence. PLOS Currents Disasters 2013; 1(Jun 5).

20. Le Roy EL, Rousseau D. Impact du climat sur la mortalité en France, de 1680 à l'époque actuelle. La Météorologie. 2009; 64:43-53.

21. Mitterstein MR, Severo DL. Análise de variabilidade intrasazonal e interanual da precipitação no vale do Itajaí com a transformada de ondaletas. Dynamis 2007; 13(1):1-10.

22. Marengo JA, Nobre CA, Salati E, Ambrizzi T. Caracterização do clima de referência e definição das alterações climáticas para o território brasileiro ao longo do século XXI: sumário técnico. Brasília: MMA, SBF, DCBio; 2007.

23. Casey JF, Kahn JR, Rivas A. Willingness to pay for improved water service in Manaus, Amazonas, Brazil. Ecological Economics 2006; 58(2):365-372.

24. Marengo JA, Tomasella J, Alves LM, Soares WR, Rodriguez DA. The drought of 2010 in the context of historical droughts in the Amazon region. Geophysical Research Letters 2011; 38:1-5.

25. Peek-Asa C, Ramirez MR, Shoaf K, Seligson H, Kraus JF. GIS mapping of earthquake-related deaths and hospital admissions from the 1994 Northridge, California, Earthquake. Ann Epidemiol 2000; 10(1):5-13.

26. Freitas CM, Ximenes EF. Enchentes e saúde pública uma questão na literatura científica recente das causas, consequências e respostas para prevenção e mitigação. Cien Saude Colet 2012; 17(6):1601-1616 .

27. Vera C, Barange M, Dube OP, Goddard L, Griggs D, Kobysheva N, Odadag E, Pareyh S, Polovinai J, Povedaj G, Seguink B, Trenberth K. Needs Assessment for Climate Information on Decadal Timescales and Longer. Procedia Environmental Sciences 2010; 1:275-286.

28. Guha-Sapir D, Below R. The quality and accuracy of disaster data: a comparative analysis of three global data sets. Bruxelas: Provention Consortium; 2002.

29. Boulos NMK, Resch B, Crowley DN, Breslin JG, Sohn G, Burtner R, Pike WA, Jezierski E, Chuang KYS. Crowdsourcing, citizen sensing and sensor web technologies for public and environmental health surveillance and crisis management: trends, OGC standards and application examples Int J Health Geogr 2011; 10:67.

30. Honorato EJSA. The interface between public health and cyberculture. Cien Saude Colet 2014; 19(2):481485.

31. Goodchild M. Citizens as sensors: the world of volunteered geography. GeoJournal 2007; 69(4):211-221.

Artigo apresentado em 07/04/2014

Aprovado em 09/06/2014

Versão final apresentada em 11/06/2014 